\title{
Bur open Dyslipidaemia in HIV-1-infected patients receiving protease inhibitors after initial treatment with first-line-based non-nucleoside reverse transcriptase inhibitors: a cross-sectional study
}

\author{
Eric Walter Pefura Yone, ${ }^{1,2}$ André Pascal Kengne, ${ }^{3}$ Gloria Ashuntantang, ${ }^{1,4}$ \\ Awa Foueudjeu Betyoumin, ${ }^{5}$ Jeanne Ngogang ${ }^{5,6}$
}

To cite: Pefura Yone EW, Kengne AP, Ashuntantang G, et al. Dyslipidaemia in HIV-1infected patients receiving protease inhibitors after initial treatment with

first-line-based nonnucleoside reverse transcriptase inhibitors: a cross-sectional study. BMJ Open 2012;0:e001317. doi:10.1136/bmjopen-2012001317

- Prepublication history and additional material for this paper are available online. To view these files please visit the journal online (http://dx. doi.org/10.1136/bmjopen2012-001317).

Received 14 April 2012 Accepted 12 July 2012

This final article is available for use under the terms of the Creative Commons Attribution Non-Commercial 2.0 Licence; see http://bmjopen.bmj.com

For numbered affiliations see end of article

Correspondence to Dr Eric Walter Pefura Yone; pefura2002@yahoo.fr

\section{ABSTRACT}

Objectives: Lipid abnormalities associated with antiretroviral therapy in people with HIV infection are more frequent with protease inhibitors (PI)-based regimens. Whether effects extend to patients receiving a PI subsequent to failure on non-nucleoside reversetranscriptase inhibitors (NNRTI)-based regimen is still unknown. We investigated the effects of secondary treatment with a PI on the lipid profile in a group of patients with HIV infection in Cameroon.

Design: This was a cross-sectional study.

Setting: This study was carried out at the registered centre for HIV treatment of the Yaounde Jamot

Hospital in Cameroon.

Participants: Participants were consecutively recruited between November 2009 and January 2010. There were $138 \mathrm{HIV}-1$ patients on initial treatment with an NNRTI regimen and 66 HIV patients on secondary treatment with a PI for at least 12 months. Lipid abnormalities were based on the National Cholesterol Education Program, Adult Treatment Panel III criteria.

Outcome measures: Levels of lipid parameters among patients on PI and NNRTI.

Results: Median (IQR) levels (mg/dl), NNRTI-treated versus PI-treated patients were 185 (149-225) and 189 (147-244) for total cholesterol, 46 (27-66) and 42 (28-82) for high-density lipoprotein (HDL)-cholesterol, 121 (90-169) and 126.9 (71-176) for low-density lipoprotein (LDL)-cholesterol, 134 (98-174) and 138 (111-167) for triglycerides, and 4.3 (2.9-6.2) and 5.1 (2.6-7.9) for total/HDL-cholesterol ratio (all $p>0.32$ ).

The most frequent lipid abnormality in the two groups was high LDL-cholesterol (46.4\% (NNRTI) vs $54.5 \%$ $(\mathrm{PI}))$. The occurrence of lipid abnormalities was similar in the two groups (all $p>0.29$ ).

Conclusions: The use of PI does not appear to deteriorate the lipid profile of HIV patients above and beyond abnormalities induced by an unsuccessful initial treatment with NNRTI. Monitoring of lipid profile during HIV treatment regardless of the regimens would

\section{ARTICLE SUMMARY}

Article focus

- To investigate the effects of secondary treatment with a protease inhibitor on the lipid profile in a group of patients with HIV infection in Cameroon.

\section{Key messages}

- The differential contribution of antiretroviral agents to lipid abnormalities suggests that hypothetically, switching patients from first-line to second-line treatment may have deleterious effects on their lipid profile.

- Effects of protease inhibitors (PI) on lipid profile among patients receiving the PI subsequent to failure on non-nucleoside reverse-transcriptase inhibitors (NNRTI)-based regimen are still unknown.

- In this study, the use of PI does not appear to deteriorate the lipid profile of HIV patients above and beyond abnormalities induced by an unsuccessful initial treatment with NNRTI.

Strengths and limitations

- To our knowledge, such hypothesis has not yet been tested.

- This is a cross-sectional study which lacked the previous level of lipid parameters of participants.

improve timely detection and management of abnormalities, to mitigate related risks.

\section{INTRODUCTION}

Antiretroviral treatments are associated with widely described abnormal changes in the lipid profile in people with HIV infection. ${ }^{1-4}$ Although more frequent during treatment with protease inhibitors (PI), ${ }^{1} 5{ }_{5} 6$ these 
changes are also observed during treatment with stavudine and to a lesser extent with non-nucleoside reversetranscriptase inhibitors (NNRTI). ${ }^{17}$ Such changes include raised total and low-density lipoprotein-cholesterol (LDL-c), raised triglycerides (TG) and variable effects on high-density lipoprotein-cholesterol (HDL-c) levels. ${ }^{16}$

The increasing rollout of antiretroviral treatment over the last 10 years has significantly improved survival among people living with HIV. ${ }^{8}$ This, however, has been achieved at the cost of increasing resistance to first-line antiretroviral therapies. ${ }^{9}{ }^{10}$ In sub-Saharan Africa (SSA), the epicentre of HIV pandemic, the widely used first-line antiretroviral regimen, inspired by the WHO, combines two nucleoside reverse-transcriptase inhibitors (NRTI) with a NNRTI. ${ }^{9}$ PI are the compulsory components of the second-line treatment subsequent to the failure of the first-line one in this setting. ${ }^{11} 12$ The differential contribution of antiretroviral agents to lipid abnormalities suggests that hypothetically switching patients from first-line to second-line treatment in SSA may have deleterious effects on their lipid profile. However, such hypothesis has not yet been tested.

We compared the lipid profile of HIV-1 patients receiving the WHO recommended first-line therapy with that of patients on second-line therapy subsequent to firstline treatment failure.

\section{PARTICIPANTS AND METHODS}

\section{Study setting and participants}

This cross-sectional study was conducted at the registered centre for HIV treatment of the Yaounde Jamot Hospital in Cameroon. The study setting has been described in detail elsewhere. ${ }^{13}$ Participants were consecutively recruited between November 2009 and January 2010. Two groups of HIV-1-positive participants were selected. The first group included individuals who had received antiretroviral treatment for the 12 preceding months or more, based on the WHO first-line regimens or NNRTI-based regimen (NNRTI-based group). The second group included individuals who were receiving second-line antiretroviral treatment or PI-based regimen for at least 12 months after failure of the first-line regimen (PI-based group). First-line ART regimens applied to these participants combined lamivudine (3TC) and stavudine (d4T) or zidovudine (AZT) with nevirapine (NVP) or efavirenz (EFV). The PI-based regimens included abacavir (ABC), didanosine (DDI) and lopinavir/ritonavir (LPV-r) or indinavir (IDV). The choice of regimens is unrelated to potential factors that could induce a dyslipidaemia, since lipid profile assessment is a requirement in routine pre-ART treatment evaluation in this setting. ${ }^{14}$ Failure on first-line antiretroviral therapy in the study setting is based on persisting plasmatic HIV-1 viral load of 5000 copies/ml or greater, after a year on antiretroviral treatment. Diagnosis of therapeutic failure to the first-line ART is always confirmed after a reinforcement of the adherence for a period of 3 months. ${ }^{11}$ Patients who had had their treatment regimens changed during follow-up were excluded. Participants had to be at least 18 years of age and to have a treatment adherence rate $\geq 95 \%$. Level of adherence was assessed by verbal administration of a standard series of questions adapted from Adult AIDS clinical trials group (AACTG) adherence instruments. The $95 \%$ rate of adherence is preferable to 4-day recall data. ${ }^{15}$ Participants were also required not to be on lipidmodifying therapies at their enrolment. All participants gave their inform consent and the study was approved by the Cameroon National Ethic Committee (ref. No. 150/CNE/SE/09).

\section{Procedures}

For each participant, data were collected on the sociodemographic background, medical history including the use of medications that could modify the lipid profile and active or history of tuberculosis. Lymphocytes count for all participants used flux cytometry methods implemented with BD FASCOUNT automate (BD Biosciences, Le pont de Claix, France). Lipid profile was assessed through enzymatic methods (Linear Chemicals, Montgat, Spain) and included total cholesterol (TC), HDL-c, LDL-c and TG. To this end, blood sample was collected after an overnight fast $(12 \mathrm{~h})$ and centrifuged at 3000 cycles/min, and the serum obtained was then used for lipid determination. The TC/HDL-c ratio was also calculated. Abnormal lipid profile was defined in accordance with the US National Cholesterol Education Program, Adult Treatment Panel III (NCEP-ATP III) guidelines, as $\mathrm{TC} \geq 200 \mathrm{mg} / \mathrm{dl}, \quad$ HDL-c $<40 \mathrm{mg} / \mathrm{dl}, \quad$ LDL-c $\geq 130 \mathrm{mg} / \mathrm{dl}$, $\mathrm{TG} \geq 150 \mathrm{mg} / \mathrm{dl}$ and TC/HDL-c ratio $\geq 5$. $^{16}$

\section{Statistical analysis}

Sample size was determined assuming a $35 \%$ prevalence of $\mathrm{TC} \geq 200 \mathrm{mg} / \mathrm{dl}$ in the NNTI-based group, a minimum detectable unadjusted OR of 2, a Type I error of $5 \%$ and a power of $80 \% .{ }^{17}$ Based on the above, the required sample size was 84 participants (42 NNTI-treated patients and 42 PI-treated patients). Data analysis used the Statistical package for social sciences (SPSS) V.17 for Windows (SPSS, Chicago, Illinois, USA). Differences in means and proportions for participants' characteristics were assessed using Student's t-test, Mann-Whitney U test and $\chi^{2}$ tests. A probability threshold of $\mathrm{p}<0.05$ was used to characterise statistical significant results.

\section{RESULTS}

\section{Study population}

The total number of participants included in each study group was 138 (NNRTI-based regimen) and 66 (PI-based regimen). NNRTI-based regimens were $\mathrm{d} 4 \mathrm{~T} /$ 3TC/NVP (61 patients), d4T/3TC/EFV (32 patients), $\mathrm{AZT} / 3 \mathrm{TC} / \mathrm{NVP}$ (31 patients) and AZT/3TC/EFV (14 patients), while PI-based regimens were $\mathrm{DDI} / \mathrm{ABC} / \mathrm{INV}$ (35 patients) and DDI/ABC/LPV-r (31 patients). 
Table 1 Profile of 138 patients on NNRTI-based regimens and 66 patients on protease inhibitors-based regimens

\begin{tabular}{|c|c|c|c|}
\hline Characteristics & NNRTI-based regimen $(n=138)$ & PI-based regimen $(n=66)$ & p Value \\
\hline Women, n (\%) & $90(65.2)$ & $39(59.1)$ & 0.396 \\
\hline Mean age, years (SD) & $40.6(8.7)$ & $42.5(8.7)$ & 0.154 \\
\hline Mean body mass index, $\mathrm{kg} / \mathrm{m}^{2}$ (SD) & $24.6(4.7)$ & $23.8(3.9)$ & 0.208 \\
\hline Median CD4 count, per mm (IQR) & $333(206-459)$ & $330(172-451)$ & 0.747 \\
\hline Median duration on ART, months (IQR) & $28(13-40)$ & $36(24-50)$ & 0.001 \\
\hline Median duration on NNRTI-based ART, months (IQR) & $28(13-40)$ & $22(10-36)$ & 0.001 \\
\hline Median duration on PI-based ART, months (IQR) & NA & $14(12-20)$ & NA \\
\hline
\end{tabular}

The characteristics of participants are summarised in table 1. The median duration on ART was higher among participants in the PI-regimen group than among those on NNRTI regimens (36 vs 28 months, $\mathrm{p}=0.001$ ). This included a 22-month period on NNRTI regimens and 14-month duration on PI-based regimens. Sex distribution, mean age, body mass index and median CD4 count were similar between the two study groups (table 1).

\section{Lipid profile}

Median levels of TC, HDL-c, LDL-c, TG and lipid ratio were similar between participants on PI-based regimens and those on NNRTI-based regimens (table 2). The prevalence of lipid abnormalities (NNRTI-based vs PI-based regimens) was $38 \%$ versus $44 \%$ for $\mathrm{TC} \geq 200 \mathrm{mg} / \mathrm{dl}$ $(p=0.39), \quad 46 \%$ versus $56 \%$ for $L D L-c \geq 130 \mathrm{mg} / \mathrm{dl}$ $(p=0.30), 40 \%$ versus $46 \%$ for HDL-c $<40 \mathrm{mg} / \mathrm{dl}(\mathrm{p}=0.54)$, 44 versus $36 \%$ for $\mathrm{TG} \geq 150 \mathrm{mg} / \mathrm{dl}(\mathrm{p}=0.33)$ and $36 \%$ versus $29 \%$ for TC/HDL-c $\geq 5 \quad(p=0.34)$. The pattern was similar in men and women, participants above and below median age, and after adjustment for age, sex and total duration on ART in linear and logistic regression analyses.

Among patients on NNRTI, median (IQR) levels (mg/ dl) of lipid variables, d4T-treated versus AZT-treated patients were 180.3 (149.2-227.1) and 227.3 (187.7270.7), $\mathrm{p}=0.632$ for total cholesterol, 133.1 (96.4-167.7) and 136.0 (136.0-101.5-189.3), $\mathrm{p}=0.389$ for $\mathrm{TG}, 46.6$ (27-67.2) and 45.2 (26.3-59.8), $\mathrm{p}=0.624$ for HDL-c, 116.8 (87.7-163.7) and 134.9 (90.6-172.7), $\mathrm{p}=0.280$ for LDL-c and $4.1(2.5-5.9)$ and $4.6(3.3-6.9), \mathrm{p}=0.341$ for total cholesterol/HDL-c ratio.
Among patients on protease inhibitors, median (IQR) levels $(\mathrm{mg} / \mathrm{dl})$ levels of lipid variables, IDV-treated versus LPV/r-treated patients were (171.9 (146.8-253.0) vs 193.8 (146.0-239.8), $\mathrm{p}=0.959)$ for TC, (135.2 (108.2-167.4) and 138.6 (112.4-162.2), $\mathrm{p}=0.96)$ for $\mathrm{TG}, 45.1$ (32.1-60.1) and 39.6 (24.0-53.8), $\mathrm{p}=0.23$ for HDL-c, 114.7 (54.4-176.7) and 130.5 (85.6-171.5), $\mathrm{p}=0.64$ for LDL-c and 4.8 (2.4-7.6) and 5.5 (2.9-8.0), $\mathrm{p}=0.576$ for TC/HDL-c ratio.

\section{DISCUSSION}

There is abundant literature on the improvement of lipid profile of people with HIV subsequent substitution of NNRTI or NRTI for PI in the treatment regimens. ${ }^{18}$ We are not aware of a study that has examined the effects of PI on lipid profile of patients initially treated with NNRTI. The current study was conducted in a setting where virtually all treated HIV-1 patients are almost always started on an NNRTI-based regimen as a first-line therapy, and shifted to PI-based regimens only after failure of the first-line therapy. Our findings suggest that this transition to PI-based regimens may not be associated with significant deterioration of the lipid profile. ${ }^{7}$ Indeed, median serum lipid levels and prevalence of lipid abnormalities were similar in patients on initial treatment with an NNRTI regimen and those on secondary treatment with a PI regimen. These findings were consistent by gender, age and CD4 count, and robust to adjustment for several covariates.

In sub-Saharan and other developing countries, NNRTI-based regimens not containing a PI are associated with pro-atherogenic adverse lipid changes. ${ }^{17}$ 19-21 However,

Table 2 Median levels of different lipid parameters in patients on NNRTI-based and PI-based regimens in Yaounde, Cameroon

\begin{tabular}{lccl}
\hline Lipid variable & NNRTI-based regimen $(\mathbf{n = 1 3 8 )}$ & PI-based regimen $(\mathbf{n}=66)$ & $\mathbf{p}$ Value \\
\hline Total cholesterol, $\mathrm{mg} / \mathrm{dl}$ & $184.7(149.3-225.0)$ & $189.5(146.7-243.8)$ & 0.688 \\
HDL cholesterol, $\mathrm{mg} / \mathrm{dl}$ & $45.84(27.0-66.0)$ & $42.16(27.6-82.3)$ & 0.568 \\
LDL cholesterol, $\mathrm{mg} / \mathrm{dl}$ & $121.4(89.7-168.7)$ & $126.9(70.8-176.5)$ & 0.767 \\
Triglycerides, $\mathrm{mg} / \mathrm{dl}$ & $134.5(98.3-174.0)$ & $5.1(2.6-7.9)$ & 0.468 \\
Total/HDL-cholesterol ratio & $4.3(2.9-6.2)$ & & 0.329 \\
\hline Data are median (IQR). & &
\end{tabular}


the WHO's recommendations, ${ }^{11} 12$ enforced at the country level in Cameroon ${ }^{14}$ and elsewhere, suggest that only those HIV patients on a PI should be screened for dyslipidaemia. These recommendations are applied against a background of scarcity of comparative studies on lipid profile of patients and NNRTI with that of those on a PI. By demonstrating findings suggesting that continuation treatment with a PI may not necessarily induce lipid derangements above and beyond those induced by prior NNRTI, our study emphasises the need to extend screening for dyslipidaemia in this setting to any patient on antiretroviral therapy regardless of the regimens. Such an approach may help in the early detection of lipid abnormalities and mitigation of related risks.

High LDL-c was the most common lipid abnormality found in our study. High LDL-c is a major lipid abnormality, and a treatment target for cardiovascular prevention. ${ }^{22}$ That lipid abnormalities in the general population in Cameroon and other countries in the region are generally rare suggests that lipid abnormalities in our patients were induced by antiretroviral therapy. It is also possible that high LDL-c was at least in part the result of the 'catch-up phenomenon'. Indeed, untreated HIV infection is associated with low LDL-c, ${ }^{23-25}$ and levels generally increase subsequent to starting antiretroviral therapy. The derangements of the lipid profile were similar in our patients treated with the regimen containing IDV or LPV-r. Likewise, the levels of lipid fractions were similar between patients receiving d4T-based regimens and those receiving AZT-based regimens. Thus, d4T does not seem to induce more dyslipidaemia in our patients as described in other studies. ${ }^{1}$ In this study, HDL-c level was similar between patients treated with NNRTI and those secondary treated with PI. Thus, switching of NNRTI regimens to PI regimens was not associated with increase of the HDL-c level. However, the results of the SMART study have shown that the use of PI is associated with a decrease of HDL-c level compared with the use of NNRTI, resulting in a higher atherogenic risk in patients treated with PI. ${ }^{26}$

TC/HDL-c ratio, a commonly used indicator of the atherogenic potential of the lipid profile ${ }^{27}$ was similar between patients on a PI and those on NNRTI regimens. This suggests that, at least in the medium term, treatment with a PI does not deteriorate the atherogenic potential of the lipid profile of patients previously on NNRTI-based regimens.

Our study has some limitations. The cross-sectional design precludes any inference about causality. It is unlikely that patients who failed on first-line antiretroviral therapy in this setting are those with a favourable lipid profile, and that our findings may actually reflect subsequent deterioration, causing their profile to be similar to that of a broader population on NNRTI. Indeed, our previous investigations in this setting suggest that markers of disease severity such as CD4 counts are not associated with lipid abnormalities. ${ }^{19}$ It is also possible that with a much large sample, some of the small differences could become significant. This, however, would not invalidate the conclusions from the study. Previous studies have demonstrated lipid abnormalities to be more frequent in NNRTI than in ART-naive patients in this setting, ${ }^{19}$ precluding the need for an ART-naive control group in the present study. Our study also has major advantages, for instance, by demonstrating perhaps for the first time that transition from an NNRTI-based regimen to a PI-based regimen should not necessarily invite changes in the monitoring protocol for lipid abnormalities.

In conclusion, treatment of HIV-1 patients with PI-based regimens subsequent to failure on NNRTI-based regimens may not deteriorate the lipid profile above and beyond derangements caused by prior NNRTI in our setting. Recommendations for lipid abnormalities in HIV patients on treatment should apply to everyone on antiretroviral treatment in this setting. Cohort data are needed to refine the findings from this study and monitor the adverse consequence of lipid derangements.

\section{Author affiliations}

${ }^{1}$ Department of Internal Medicine and Subspecialties, Faculty of Medicine and Biomedical Sciences, University of Yaounde 1, Yaounde, Cameroon

${ }^{2}$ Service of Pneumology, Yaounde Jamot Hospital, Yaounde, Cameroon

${ }^{3}$ South African Medical Research Council \& University of Cape Town, Cape Town, South Africa

${ }^{4}$ Service of Internal Medicine, Yaounde General Hospital, Yaounde, Cameroon ${ }^{5}$ Department of Biochemistry, Yaounde University Centre, Yaounde, Cameroon ${ }^{6}$ Department of Biochemistry, Faculty of Medicine and Biomedical Sciences, University of Yaounde 1, Yaounde, Cameroon

Contributors EWPY conceived the study, supervised data collection, co-analysed the data and drafted of the manuscript; APK contributed to study designed, data analysis, drafting and critical revision of the manuscript; GA contributed to study design and critically revised the manuscript; AFB contributed to data collection, co-analysed the data and drafting of the manuscript; JN supervised data collection and critically revised the manuscript. All authors approved the final version of the manuscript.

Competing interests None.

Data sharing statement No additional data are available.

\section{REFERENCES}

1. Grunfeld C. Dyslipidemia and its treatment in HIV infection. Top HIV Med 2010;18:112-18.

2. Troll JG. Approach to dyslipidemia, lipodystrophy, and cardiovascular risk in patients with HIV infection. Curr Atheroscler Rep 2011;13:51-6.

3. Haubrich RH, Riddler SA, DiRienzo AG, et al. Metabolic outcomes in a randomized trial of nucleoside, nonnucleoside and protease inhibitor-sparing regimens for initial HIV treatment. AIDS 2009;23:1109-18.

4. Friis-Moller N, Sabin CA, Weber R, et al. Combination antiretroviral therapy and the risk of myocardial infarction. $N$ Engl J Med 2003;349:1993-2003.

5. Heath KV, Chan KJ, Singer J, et al. Incidence of morphological and lipid abnormalities: gender and treatment differentials after initiation of first antiretroviral therapy. Int J Epidemiol 2002;31:1016-20.

6. Feeney ER, Mallon PW. HIV and HAART-associated dyslipidemia. Open Cardiovasc Med J 2011;5:49-63.

7. Domingos H, Cunha RV, Paniago AM, et al. Metabolic effects associated to the highly active antiretroviral therapy (HAART) in AIDS patients. Braz J Infect Dis 2009;13:130-6.

8. Palella FJ Jr., Delaney KM, Moorman AC, et al. Declining morbidity and mortality among patients with advanced human immunodeficiency virus infection. HIV outpatient study investigators. N Engl J Med 1998;338:853-60. 
9. Gsponer T, Petersen M, Egger M, et al. The causal effect of switching to second-line ART in programmes without access to routine viral load monitoring. AIDS 2012;26:57-65.

10. von Wyl V, Yerly S, Boni J, et al. Incidence of hiv-1 drug resistance among antiretroviral treatment-naive individuals starting modern therapy combinations. Clin Infect Dis 2012;54:131-40.

11. Organisation Mondiale de la Santé. Recommandations rapides: Traitement antirétroviral de l'infection à VIH chez l'adulte et l'adolescent 2010. http://www.who.int/hiv/pub/arv/ rapid_advice_art_fr.pdf (accessed 12 Dec 2011).

12. Ministère de la Santé Publique du Cameroun. Directives nationales de prise en charge par les antirétroviraux des personnes (adultes et adolescents) infectés par le VIH. Yaoundé 2010.

13. Pefura Yone EW, Kengne AP, Kuaban C. Incidence, time and determinants of tuberculosis treatment default in Yaounde, Cameroon: a retrospective hospital register-based cohort study. BMJ Open 2011;1:e000289.

14. World Health Organization. Antiretroviral therapy for HIV infection in adults and adolescents in resource-limited settings: towards universal access. 2006 revision http://www.who.int/hiv/pub/arv/adult/ fr/index.html (accessed 10 Oct 2010).

15. Chesney MA, Ickovics JR, Chambers DB, et al. Self-reported adherence to antiretroviral medications among participants in HIV clinical trials: the AACTG adherence instruments. Patient care committee \& adherence working group of the outcomes committee of the Adult Aids Clinical Trials Group (AACTG). AIDS Care 2000;12:255-66.

16. National Cholesterol Education Program (NCEP) expert panel on detection, evaluation, and treatment of high blood cholesterol in adults (Adult Treatment Panel III) final report. Circulation 2002;106:3143-421.

17. Zannou DM, Denoeud L, Lacombe K, et al. Incidence of lipodystrophy and metabolic disorders in patients starting non-nucleoside reverse transcriptase inhibitors in benin. Antivir Ther 2009;14:371-80.

18. Lake JE, Currier JS. Switching antiretroviral therapy to minimize metabolic complications. HIV Ther 2010;4:693-711.

19. Pefura Yone EW, Betyoumin AF, Kengne AP, et al. First-line antiretroviral therapy and dyslipidemia in people living with HIV-1 in Cameroon: a cross-sectional study. AIDS Res Ther 2011;8:33.

20. Buchacz K, Weidle PJ, Moore D, et al. Changes in lipid profile over 24 months among adults on first-line highly active antiretroviral therapy in the home-based AIDS care program in rural Uganda. $J$ Acquir Immune Defic Syndr 2008;47:304-11.

21. Padmapriyadarsini C, Ramesh Kumar S, Terrin N, et al. Dyslipidemia among HIV-infected patients with tuberculosis taking once-daily nonnucleoside reverse-transcriptase inhibitor-based antiretroviral therapy in India. Clin Infect Dis 2011;52:540-6.

22. Grundy SM, Cleeman JI, Merz CN, et al. Implications of recent clinical trials for the national cholesterol education program adult treatment panel iii guidelines. J Am Coll Cardiol 2004;44:720-32.

23. Oka F, Naito T, Oike M, et al. Correlation between HIV disease and lipid metabolism in antiretroviral-naive HIV-infected patients in Japan. $J$ Infect Chemother 2011;18:17-21.

24. Constans J, Pellegrin JL, Peuchant E, et al. Plasma lipids in HIV-infected patients: a prospective study in 95 patients. Eur J Clin Invest 1994;24:416-20.

25. Riddler SA, Smit E, Cole SR, et al. Impact of HIV infection and HAART on serum lipids in men. JAMA 2003;289:2978-82.

26. Phillips AN, Carr A, Neuhaus $\mathrm{J}$, et al. Interruption of antiretroviral therapy and risk of cardiovascular disease in persons with HIV-1 infection: exploratory analyses from the SMART trial. Antivir Ther 2008;13:177-87.

27. Hsia SH, Pan D, Berookim P, et al. A population-based, cross-sectional comparison of lipid-related indexes for symptoms of atherosclerotic disease. Am J Cardiol 2006;98:1047-52. 